\title{
Das besondere Buch
}

\author{
Erhard Taverna
}

Das Werk ist nicht geeignet, im Kittel mitgeführt oder auf dem Rücken liegend im Bett gelesen zu werden. Es ist keine Checkliste, kein Leitfaden und schon gar kein Kochbuch. Die 1261 Seiten sind Bibliothek und Fundgrube, ein konzises Kompendium und Protokoll einer jahrzehntelangen Berufserfahrung. Und ausserdem ein ganz besonderes Lesevergnügen, weil der doppelt begabte Autor sein reiches klinisches Wissen mit einer didaktischen Begabung vereinigt.

Die Rede ist von Alfred M. Debrunner und der 4. vollständig neu bearbeiteten Auflage seiner «Orthopädie - Orthopädische Chirurgie.» Als derart umfangreiches «Einmannbuch» steht es einsam da im Markt der schnellen Nachschlagewerke und der unabsehbaren Flut rascher Publikationen, die weniger den Leser als vielmehr den Zitationsindex im Auge haben. «Ich habe versucht, ein Buch für die praktische ärztliche Arbeit für und mit dem Patienten zu schreiben. Dieser soll im Mittelpunkt stehen. Daher der Untertitel: ‘Patientenorientierte Diagnostik und Therapie des Bewegungsapparates . Ich habe mich um eine leicht lesbare, einfache Sprache bemüht, so wie wir Ärzte auch mit den Patienten reden sollten.» Der Autor schreibt für Allgemeinpraktiker und Ärzte, die Menschen beider Geschlechter und aller Lebensalter in ihrer Praxis betreuen. Störungen des Bewegungsapparates dominieren den Alltag des Familienarztes von der Hüftkontrolle des Neugeborenen, über die Knieschmerzen des Sportverletzten bis zum Schenkelhalsbruch bei fortgeschrittener Osteoporose. Leser und Leserin haben die seltene Gelegenheit, das Lehrbuch eines Spezialisten in der Hand zu haben, der weit über seine Fachgrenzen hinaus Verbindliches zu sagen hat. Er gehört nicht zu jener Kategorie Kollegen, auf die das Zitat aus dem Buch zutrifft: «If the only tool you have is a hammer, you tend to see every problem as a nail.» Debrunner vermittelt eine Darstellung von Prinzipien, ein Raster von Basiswissen, eine Matrix, in die neue Erkenntnisse sinnvoll eingeordnet werden können.

Dazu ist der Band in drei Teile gegliedert: einen ersten zur Biomechanik, Pathophysiologie und Diagnostik, einen zweiten zur allgemeinen Orthopädie und einen dritten Teil zur regionalen Orthopädie von Wirbelsäule und Extremitäten. Spezielle Einschübe wie die Kapitel über

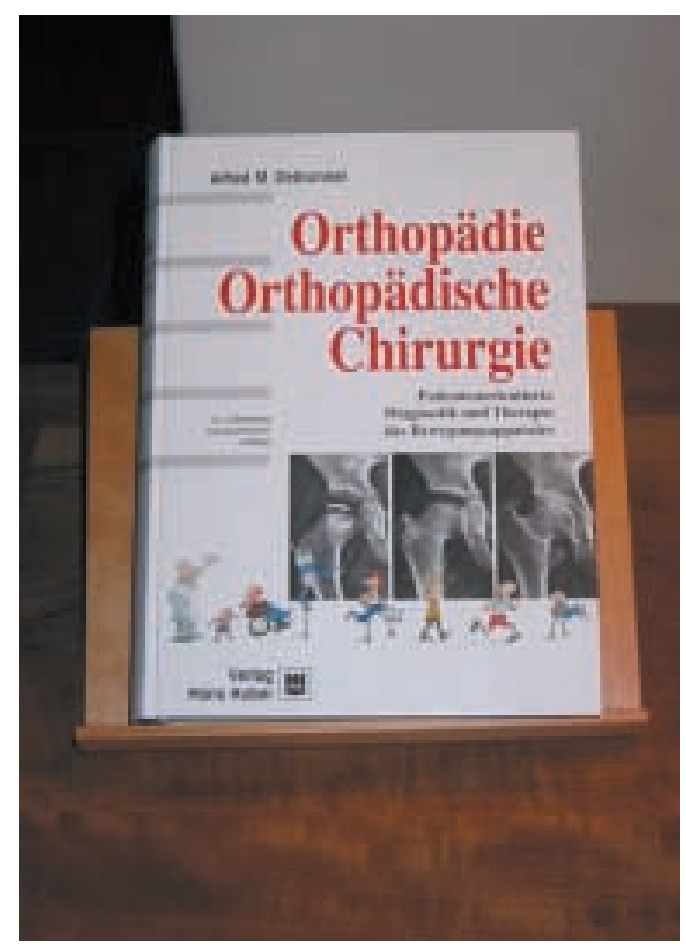

«Prinzipien, Methoden und Indikationen» oder «Psychosomatik» vermitteln besten interdisziplinären Unterricht, sachkundig, humorvoll und schnörkellos vorgetragen. Im Abschnitt «Orthopädie von der Geburt bis zum Tod - zwischen Patient und Wissenschaft» wagt der Autor einen Exkurs über den Faktor Zeit, über Langzeitforschung und Statistik und damit über die Besonderheiten der Sport- und Kinderchirurgie. Ihm gelingt es immer wieder, Aspekte einzubringen, die in der orthopädischen Fachliteratur selten untersucht werden: zum Beispiel die Ansprüche des Kranken auf Information, seinen Entscheidungsprozess und dessen Grenzen, Langzeitforschung, Selbstkritik und pekuniäre Chancen und Risiken der Operateure bis hin zum industriellen Sponsoring. Doch Hauptanliegen bleibt die Lehre des notwendigen Fachwissens, das sprachlich vorbildlich, mit Hilfe instruktiver Bilder, gut ausgesuchter Graphiken und mit praktischen Querverweisen vermittelt wird. Zudem wurden selten noch Zitate in einem Lehrbuch derart humorvoll und bereichernd eingesetzt. Eine Bibliographie mit Gebrauchsanweisung erschliesst den Zugang zu weiterführender Literatur. 
Gemäss einer brieflichen Mitteilung wollte Alfred M. Debrunner diese 4. Auflage eigentlich nicht mehr schreiben. Er habe weder in der Schweiz noch unter seinen deutschen Kollegen jemanden gefunden, der sich dafür interessiert habe. Lehrbücher brächten eben keine Lorbeeren und alle hätten Wichtigeres und Dringlicheres zu tun gehabt.

Debrunner hat viel gewagt und viel gewonnen. Darum seien ihm Studenten, Ärztinnen und Ärzte vergönnt, auf die das zutrifft, was
Brecht einst über das Werk von Laotse bemerkte: «Aber rühmen wir nicht nur den Weisen - Dessen Name auf dem Buche prangt! - Denn man muss dem Weisen seine Weisheit erst entreissen. - Darum sei der Zöllner auch bedankt: - Er hat sie ihm abverlangt.»

Alfred M. Debrunner. Orthopädie - Orthopädische Chirurgie. 4. vollständig neu bearbeitete Auflage. Bern: Verlag Hans Huber; 2002.

\section{Der Wandel der Ansprüche an den Arzt}

\section{A. M. Debrunner}

Korrespondenz:

Dr. med. Alfred M. Debrunner

Mettlenstrasse 15

CH-8142 Uitikon
Dieser Wandel zeigt sich drastisch bereits sprachlich, indem der Arzt zuerst zum Mediziner und inzwischen zum «Leistungserbringer» verwandelt wurde, von dem nicht mehr Beratung, Begleitung und Hilfe in der Not erwartet wird, sondern technische Reparatur von Gesundheitsdefiziten, wo doch Anspruch auf intakte Gesundheit bereits als ein Menschenrecht gilt.

Dass diese Vorstellung eine Utopie ist, zeigt sich für jeden Bürger deutlich sichtbar in der «Kostenexplosion» im Gesundheitswesen, die nichts anderes ist als die "Leistungsexplosion» eines Sisyphus, der letztlich, den Tod zu bezwingen, sich zum Ziel gesetzt hat. Dass das nicht funktionieren kann, wäre zwar einsichtig, aber wahrhaben will es niemand. Die sich daraus ergebenden Konsequenzen sind für die meisten inakzeptabel und deshalb tabu. Als da wären: Rationierung oder Mehrklassenmedizin, beides Horrorszenarien. Oder aber: Man könnte ohne weiteres statt 12 oder 15 oder $20 \%$ auch 90\% des BSP ins Gesundheitswesen stecken, wie Prof. Ölz gesagt hat, wenn man wirklich davon überzeugt ist, dass Gesundheit das höchste Gut sei.

Was hat die Verfassung des schweizerischen Gesundheitswesens mit medizinischen Lehrbüchern zu tun? Ich selbst habe die Entwicklung der Medizin in den letzten 60 Jahren ziemlich gut mitverfolgen können. Mein Vater war schon Orthopäde, und ich durfte ihm bei den ersten Hüftoperationen helfen. Der Weg, den die Medizin seither gemacht hat, ist tatsächlich imponierend. Paradox erscheint hingegen, dass das Gesundheitswesen in der Zeit nach dem zweiten Weltkrieg eigentlich recht gut funktionierte, jedenfalls waren alle ziemlich zufrieden damit: Patienten, Ärzte, Spitäler, Apotheker, Therapeuten, die Industrie, die Politiker, die Krankenkassen, die Versicherten und die Steuerzahler. Doch umgekehrt proportional zur Erhöhung des Angebotes und zur Verbesserung der Dienstleistungen stieg die Unzufriedenheit.

Ich bin überzeugt, dass unser schweizerisches Gesundheitswesen eines der besten ist und auf einem sehr hohen Niveau steht, und dass dieses erhalten werden sollte. Ich glaube aber auch, dass eine ausschliesslich technische Betrachtungsweise, wie sie heute von zwei Seiten kommt, nicht ausreicht, dieses zu retten: Die eine Seite ist diejenige der Ökonomen, in deren eindimensionaler Sicht wir als Leistungserbringer auf Zahlen reduzierbar sind. Die andere Seite ist die ärztliche, und diese können wir beeinflussen, vielleicht sogar mit meinem Lehrbuch. Denn fatal wirkt sich vor allem die Einmütigkeit von Ärzten und Publikum im Glauben an die technische Machbarkeit von Gesundheit aus, eine eigentliche «folie à deux», gegen die nur wir selbst Gegensteuer geben können. 